\title{
Multivalued Fuzzy Logics: A Sensitive Analysis
}

\author{
MsC. Ing. Pablo Michel Marin Ortega ${ }^{1}$, Dr. Rafael Espín Andrade², Dr.-Ing. habil. Jorge Marx Gómez ${ }^{3}$ \\ ${ }^{1}$ University of Las Villas, Santa Clara, Cuba \\ ${ }^{2}$ José Antonio Echeverría" (CUJAE) University, La Habana, Cuba \\ ${ }^{3}$ Carl von Ossietzky University Oldenburg, Oldenburg, Germany \\ pablomo@uclv.edu.cu, espin@ind.cujae.edu.cu,jorge.marx.gomez@uni-oldenburg.de
}

\begin{abstract}
The main goal of this research is develop a sensitive analysis (SA) among some fuzzy operators, to ask the question: which is the most robustness fuzzy operators? The fuzzy operators consider in this study are: Zadeh operators, Probabilistic operators and finally the compensatory fuzzy logic operators: Geometric mean and Arithmetic mean. The Sobol model and the Monte Carlo simulations was been used to develop the SA. According with the main result of the study the compensatory fuzzy logic operators are the most robust.
\end{abstract}

Keywords: Sensitive analysis, Sobol model and the Monte Carlo simulations, fuzzy operators.

\section{Introduction}

Nowadays the fuzzy operators are widely used in the decision making field (Merigó and Gil-Lafuente, 2011, Wei and Zhao, 2012, Zandi and Tavana, 2012, Cebeci, 2009, Yüksel and Dağdeviren, 2010) because some of their essential properties including facilities to model the "vagueness" proper to the natural language and the uncertainty. Based on this stronghold the business analyst can builds a semantic model and then they can use the inference capability of the logical platform to facilitate assessment of any situation, decision making or knowledge discovery.

However in the current literature are several fuzzy operators to do it. The most common operators are: Zadeh(Zadeh, 1973, Dubois and Prade, 1985), Probabilistic / Algebraic(Dubois and Prade, 1985), Geometric Mean based Compensatory Logic (Espin Andrade et al., 2003) and finally Arithmetic Mean based Compensatory Logic(Bouchet et al., 2010), based on it the primary question is: which is the most robustness operators according with the problem to resolve?

The SA study how uncertainty in the output of a model (numerical or otherwise) can be apportioned to different sources of uncertainty in the model input(Saltelli et al., 2004). A related practice is uncertainty analysis, which focuses rather on quantifying uncertainty in model output. Ideally, uncertainty and sensitivity analyses should be run in tandem, with uncertainty analysis preceding in current practice(Saltelli et al., 2008).

The main goal of this research is make a SA among the basic expression used in the fuzzy logic field versus the fuzzy operators mentioned above, to do it the paper was organized as follows: Section 2 provides a description of the fuzzy operators. In Section 3, we present important outlines about SA methods. Section 4 we describe the methodology used to develop the SA. The results are discussed in Section 5. Finally, conclusions and future work are mentioned in Section 6.

\section{Fuzzy Operators}

The operators for the logic connective: conjunction ("and") and disjunction ("or") can be group by operators models. The operator models that we use in this research and their mathematics expressions are showing below.

\subsection{Max-Min(Dubois and Prade, 1985)}

- Conjunction

$$
\mathrm{C}(\mathrm{X} 1, \mathrm{X} 2, \ldots \mathrm{Xn})=\mathrm{Min}(\mathrm{X} 1, \mathrm{X} 2, \ldots \mathrm{Xn}) \ldots(2.1)
$$

- Disjunction

$\mathrm{D}(\mathrm{X} 1, \mathrm{X} 2, \ldots \mathrm{Xn})=\operatorname{Max}(\mathrm{X} 1, \mathrm{X} 2, \ldots \mathrm{Xn}) \ldots(2.2)$

\subsection{Probabilistic / Algebraic (Dubois and Prade,} 1985)

- Conjunction $C\left(X_{1}, X_{2}, \ldots X_{n}\right)=X_{1} \times X_{2} \times \ldots \times X_{n} \ldots$

- Disjunction

$$
\begin{aligned}
& D\left(X_{1}, X_{2}, \ldots X_{n}\right)=\left(1^{0}\right) \times \sum_{i=1}^{i=n} X_{i}+ \\
& \left(1^{1}\right) \times \sum_{i \neq j}^{i=n} X_{i} \times X_{j}+\left(1^{2}\right) \times \sum_{\substack{i \neq j \\
i \neq j}}^{i \neq n} X_{i} \times X_{j} \times X_{k} \\
& +\ldots+X_{1} \times X_{2} \times \ldots \times X_{n}
\end{aligned}
$$




\subsection{Geometric Mean based Compensatory Logic (GMBCL) (Espin Andrade et al., 2003)}

- Conjunction

$C\left(X_{1}, X_{2}, \ldots X_{n}\right)=\left(X_{1} \times X_{2} \times . . \times X_{n}\right)^{1 / n}$

- Disjunction

$\mathrm{D}\left(\mathrm{X}_{1}, \mathrm{X}_{2}, \ldots \mathrm{X}_{\mathrm{n}}\right)=1-\left[\left(1-\mathrm{X}_{1}\right) \times\left(1-\mathrm{X}_{2}\right) \times . . \times\left(1-\mathrm{X}_{\mathrm{n}}\right)\right]^{1 / n}$ ... (2.6)

\subsection{Arithmetic Mean based Compensatory Logic (AMBCL) (Bouchet et al., 2010)}

- Conjunction

$$
\mathrm{C}\left(\mathrm{X}_{1}, \mathrm{X}_{2}, \ldots \mathrm{X}_{\mathrm{n}}\right)=\left[\min \left(\mathrm{X}_{1} \times \mathrm{X}_{2} \times . . \times \mathrm{X}_{\mathrm{n}}\right) \times \frac{\sum_{\mathrm{i}=1}^{\mathrm{i}=\mathrm{n}} \mathrm{x}_{\mathrm{i}}}{\mathrm{n}}\right]
$$

- Disjunction

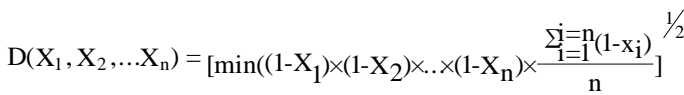

\subsection{Implication}

- $\quad$ Zadeh (Zadeh, 1973)

$$
\mathrm{A} \rightarrow \mathrm{B}=\neg \mathrm{A} \vee \mathrm{B} \ldots \text { (2.9) }
$$

- Kleene-Dienes(Piegat, 2001)

$$
\mathrm{A} \rightarrow \mathrm{B}=\neg \mathrm{A} \vee(\mathrm{A} \wedge \mathrm{B})
$$

Note: The implication is calculated based on the disjunction and conjunction operators used in the problem.

\subsection{Negation}

$$
\mathrm{F}(\mathrm{x})=1-\mathrm{X}
$$

\section{Major Headings Sensitive analysis methods}

Many SA methods are based on an analysis of the variance of the model output (Saltelli et al., 2008); the theoretical basis of several of these methods is variance decomposition. Such techniques include Fourier Amplitude Sensitivity Test (FAST), High Dimensional Model Representation (HDMR), random balance designs, and traditional ANOVA methods (Glen and Isaacs, 2012). In variance decomposition, the model output variance is represented as a sum of (2J-1) partial variances. Here, $\mathbf{J}$ is the number of random samples needed per model iteration. While $\mathrm{J}$ is often called "the number of inputs", this is not necessarily the same as the number of modeling input variables, because some models (typically ones with either spatial or temporal variation) may draw multiple samples for the same modeling variable on each iteration (Glen and Isaacs, 2012).

According with (Yang, 2011) the Sobol's method has been shown to be robust and it is superior to traditional sensitivity methods (such as local methods that examine parameters one at a time) when considering cases where the assumption of linearity is invalid(Saltelli et al., 2004). Sobol's method is a global SA technique which determines the contribution of each input (or group of inputs) to the variance of the output. The usual Sobol sensitivity indices include the main and total effects for each input, but the method can also provide specific interaction terms, if desired. The method is notable because it works well without simplifying approximations, even for models with very large numbers of random variables.

The Sobol sensitivity indices are ratios of partial variances to total variance, and for independent variables satisfy the follow relationship (Saltelli et al., 2008).

$$
\sum_{1} \mathrm{~S}_{\mathrm{i}}+\sum_{1} \sum_{\mathrm{j}>\mathrm{i}} \mathrm{S}_{\mathrm{ij}}+\sum_{1} \sum_{\mathrm{j}>\mathrm{i}} \sum_{>>\mathrm{j}} \mathrm{S}_{\mathrm{ijl}}+\ldots+\mathrm{S}_{123}=1
$$

The conditional expectation $\mathrm{E}\left(\mathrm{Y} \mid \mathrm{X}_{\mathrm{i}}\right)$ can be calculated empirically by cutting the $\mathrm{Xi}$ domain into slices and averaging the values of $\left(\mathrm{Y} \mid \mathrm{X}_{\mathrm{i}}\right)$ within the same slice $\mathrm{Xi}$. In this way, if the scatterplot has a pattern, the conditional expectation $\mathrm{E}\left(\mathrm{Y} \mid \mathrm{X}_{\mathrm{i}}\right)$ has a large variation across $\mathrm{Xi}$ values and the factor $\mathrm{Xi}$ is revealed to be important. Hence, the variance of the conditional expectation can be considered as a summary measure of sensitivity. In fact, the variances of the terms in the decomposition above are the measures of importance being sought. In particular, $\mathrm{V}(\mathrm{Fi}(\mathrm{Xi}))$ is $\mathrm{V}\left[\mathrm{E}\left(\mathrm{Y} \mid \mathrm{X}_{\mathrm{i}}\right)\right]$; when we divide this by the unconditional variance $\mathrm{V}(\mathrm{Y})$, we obtain the first-order sensitivity index. In short (Saltelli et al., 2008):

$\mathrm{S}_{\mathrm{i}}=\frac{\mathrm{V}\left[\mathrm{E}\left(\mathrm{Y} \mid \mathrm{X}_{\mathrm{i}}\right)\right]}{\mathrm{V}(\mathrm{Y})}$

Sobol' also proposed a comparable definition of $\mathrm{Si}$ which is based on the correlation between the model $\mathrm{Y}$ and the conditional expectation $\mathrm{E}\left(\mathrm{Y} \mid \mathrm{X}_{\mathrm{i}}\right)$ (Saltelli et al., 2008):

$\mathrm{S}_{\mathrm{i}}=\operatorname{Corr}\left[\mathrm{Y}, \mathrm{E}\left(\mathrm{Y} \mid \mathrm{X}_{\mathrm{i}}\right)\right]$

The first-order index $\mathrm{Si}$ represents the main effect contribution of each input factor to the variance of the output.

The total effect index St accounts for the total contribution to the output variation due to factor $\mathrm{Xi}$, its first-order effect plus all higher-order effects due to interactions.

The total effect measure provides the educated answer to the question: Which factor can be fixed anywhere over its range of variability without affecting the output? The condition $\mathrm{Sti}=0$ is necessary and sufficient for $\mathrm{Xi}$ 
to be a noninfluential factor. If $\mathrm{S}_{\mathrm{ti}} \approx 0$, then $\mathrm{Xi}$ can be fixed at any value within its range of uncertainty without appreciably affecting the value of the output variance $\mathrm{V}(\mathrm{Y})$. The approximation error that is made when this model simplification is carried out depends on the value of Sti. Total indices are suitable for the factor fixing setting (Saltelli et al., 2008).

The measure $\mathrm{V}(\mathrm{Y})-\mathrm{V}(\mathrm{E}(\mathrm{Y} \mid \mathrm{X} \sim \mathrm{i}))=\mathrm{E}(\mathrm{V}(\mathrm{Y} \mid \mathrm{X} \sim \mathrm{i}))$ is the remaining variance of $\mathrm{Y}$ that would be left, on average, if we could determine the true values of $\mathrm{X} \sim \mathrm{i}$. The average is calculated over all possible combinations of $\mathrm{X} \sim \mathrm{i}$, since $\mathrm{X} \sim \mathrm{i}$ are uncertain factors and their true values are unknown. Dividing by $\mathrm{V}(\mathrm{Y})$ we obtain the total effect index for $\mathrm{Xi}$ (Saltelli et al., 2008):

$\mathrm{S}_{\mathrm{ti}}=\frac{\mathrm{E}\left[\mathrm{V}\left(\mathrm{Y} \mid \mathrm{X}_{\mathrm{i}}\right)\right]}{\mathrm{V}(\mathrm{Y})}=1-\frac{\mathrm{V}\left[\mathrm{E}\left(\mathrm{Y} \mid \mathrm{X}_{\mathrm{i}}\right)\right]}{\mathrm{V}(\mathrm{Y})}$

Table 1: Expressions evaluated in the research

\begin{tabular}{|l|l|l|l|}
\hline $\mathrm{N}$ & Expression & $\mathrm{N}$ & Expression \\
\hline 4.1 & $\mathrm{~A} \cap \mathrm{B}$ & 4.7 & $\mathrm{C} \cap(\mathrm{A} \cap \mathrm{B})$ \\
\hline 4.2 & $\mathrm{~A} \cup \mathrm{B}$ & 4.8 & $\mathrm{C} \cup(\mathrm{A} \cup \mathrm{B})$ \\
\hline 4.3 & $\mathrm{~A} \rightarrow \mathrm{B}$ (Zadeh) & 4.9 & $\begin{array}{l}(\mathrm{A} \rightarrow \mathrm{B}) \cap(\mathrm{C} \rightarrow \mathrm{D}) \\
(\text { Kleene-Dienes })\end{array}$ \\
\hline 4.4 & $\begin{array}{l}\mathrm{A} \rightarrow \mathrm{B}(\text { Kleene- } \\
\text { Dienes })\end{array}$ & 4.10 & $\begin{array}{l}(\mathrm{A} \rightarrow \mathrm{B}) \cup(\mathrm{C} \rightarrow \mathrm{D}) \\
(\text { Kleene-Dienes })\end{array}$ \\
\hline 4.5 & $\mathrm{C} \cup(\mathrm{A} \cap \mathrm{B})$ & 4.12 & $\begin{array}{l}(\mathrm{A} \rightarrow \mathrm{B}) \cap(\mathrm{C} \rightarrow \mathrm{D}) \\
(\text { Zadeh })\end{array}$ \\
\hline 4.6 & $\mathrm{C} \cap(\mathrm{A} \cup \mathrm{B})$ & 4.13 & $\begin{array}{l}(\mathrm{A} \rightarrow \mathrm{B}) \cup(\mathrm{C} \rightarrow \mathrm{D}) \\
(\text { Zadeh })\end{array}$ \\
\hline
\end{tabular}

Table 2: Output of the SA

\begin{tabular}{|c|c|c|c|c|c|c|}
\hline Number & Exp/Ope & & Zadeh & Probabilístico & GMBCL & AMBCL \\
\hline \multirow[t]{5}{*}{4.1} & $A \cap B$ & $\mathrm{a}$ & 0.461079 & 0.546975 & 0.58177 & 0.536315 \\
\hline & & $\mathrm{b}$ & 0.399166 & 0.492651 & 0.507603 & 0.46457 \\
\hline & & $a, b$ & 0.217443 & 0.059741 & 0.047327 & 0.108623 \\
\hline & & Total a & 0.701357 & 0.65139 & 0.601131 & 0.635665 \\
\hline & & Total b & 0.709885 & 0.66146 & 0.595311 & 0.635519 \\
\hline \multirow[t]{5}{*}{4.2} & $\mathrm{~A} \cup \mathrm{B}$ & $\mathrm{a}$ & 0.392874 & 0.443672 & 0.470392 & 0.452613 \\
\hline & & $\mathrm{b}$ & 0.409005 & 0.411461 & 0.431699 & 0.440581 \\
\hline & & $\mathrm{a}, \mathrm{b}$ & 0.24438 & 0.218927 & 0.14337 & 0.160309 \\
\hline & & Total a & 0.64999 & 0.602127 & 0.558783 & 0.59059 \\
\hline & & Total b & 0.572444 & 0.537669 & 0.461621 & 0.502594 \\
\hline \multirow[t]{5}{*}{4.3} & $\mathrm{~A} \rightarrow \mathrm{B}$ (Zadeh) & $\mathrm{a}$ & 0.512678 & 0.509999 & 0.579183 & 0.558674 \\
\hline & & $\mathrm{b}$ & 0.412699 & 0.469531 & 0.474317 & 0.455549 \\
\hline & & $\mathrm{a}, \mathrm{b}$ & 0.133468 & 0.078123 & 0.005043 & 0.046291 \\
\hline & & Total a & 0.622163 & 0.610358 & 0.604376 & 0.606375 \\
\hline & & Total b & 0.683242 & 0.689642 & 0.607451 & 0.632435 \\
\hline 4.4 & $\mathrm{~A} \rightarrow \mathrm{B}$ (Kleene-Dienes) & $\mathrm{a}$ & 0.633352 & 0.66973 & 0.633815 & 0.640718 \\
\hline
\end{tabular}




\begin{tabular}{|c|c|c|c|c|c|c|}
\hline Number & Exp/Ope & & Zadeh & Probabilístico & GMBCL & AMBCL \\
\hline & & $\mathrm{b}$ & 0.173623 & 0.237481 & 0.339914 & 0.25294 \\
\hline & & $a, b$ & 0.22159 & 0.148192 & 0.088994 & 0.154152 \\
\hline & & Total a & 0.839817 & 0.82402 & 0.742567 & 0.796698 \\
\hline & & Total b & 0.581495 & 0.576333 & 0.633455 & 0.592526 \\
\hline \multirow[t]{10}{*}{4.5} & $\mathrm{C} \cup(\mathrm{A} \cap \mathrm{B})$ & $\mathrm{a}$ & 0.009852 & 0.052189 & 0.118878 & 0.075455 \\
\hline & & $\mathrm{b}$ & 0.026194 & 0.099134 & 0.169561 & 0.110831 \\
\hline & & $\mathrm{c}$ & 0.672507 & 0.762482 & 0.725054 & 0.709961 \\
\hline & & $a, b$ & 0.108087 & 0.023273 & 0.025583 & 0.055324 \\
\hline & & $\mathrm{a}, \mathrm{c}$ & 0.135542 & 0.109461 & 0.08162 & 0.097641 \\
\hline & & $\mathrm{b}, \mathrm{c}$ & 0.052361 & 0.054117 & 0.024602 & 0.042089 \\
\hline & & $\mathrm{a}, \mathrm{b}, \mathrm{c}$ & -0.0228 & -0.0295 & -0.0259 & -0.0338 \\
\hline & & Total a & 0.21919 & 0.155736 & 0.225491 & 0.209213 \\
\hline & & Total b & 0.217354 & 0.160235 & 0.233487 & 0.226803 \\
\hline & & Total c & 0.768611 & 0.805313 & 0.688758 & 0.726553 \\
\hline & & & & & & \\
\hline \multirow[t]{10}{*}{4.6} & $\mathrm{C} \cap(\mathrm{A} \cup \mathrm{B})$ & $\mathrm{a}$ & 0.020657 & 0.078882 & 0.122643 & 0.075729 \\
\hline & & $\mathrm{b}$ & 0.022311 & 0.060447 & 0.102986 & 0.070118 \\
\hline & & $\mathrm{c}$ & 0.598384 & 0.724479 & 0.709126 & 0.659737 \\
\hline & & $\mathrm{a}, \mathrm{b}$ & 0.087431 & 0.043192 & 0.053118 & 0.076683 \\
\hline & & $\mathrm{a}, \mathrm{c}$ & 0.109971 & 0.061229 & 0.037376 & 0.069521 \\
\hline & & $\mathrm{b}, \mathrm{c}$ & 0.107722 & 0.088154 & 0.055905 & 0.072386 \\
\hline & & $a, b, c$ & -0.0454 & -0.053 & -0.024 & -0.0372 \\
\hline & & Total a & 0.239454 & 0.221987 & 0.248581 & 0.238633 \\
\hline & & Total b & 0.167485 & 0.119752 & 0.158304 & 0.156916 \\
\hline & & Total c & 0.795197 & 0.800222 & 0.718107 & 0.752178 \\
\hline \multirow[t]{10}{*}{4.7} & $\mathrm{C} \cap(\mathrm{A} \cap \mathrm{B})$ & $\mathrm{a}$ & 0.406425 & 0.481011 & 0.318524 & 0.360879 \\
\hline & & $\mathrm{b}$ & 0.17918 & 0.195726 & 0.204244 & 0.180705 \\
\hline & & $\mathrm{c}$ & 0.221876 & 0.138834 & 0.633334 & 0.474482 \\
\hline & & $\mathrm{a}, \mathrm{b}$ & 0.084522 & 0.021548 & 0.015215 & 0.035204 \\
\hline & & $\mathrm{a}, \mathrm{c}$ & 0.069457 & 0.052648 & -0.00823 & 0.020512 \\
\hline & & $\mathrm{b}, \mathrm{c}$ & 0.115569 & 0.10475 & 0.043139 & 0.079477 \\
\hline & & $\mathrm{a}, \mathrm{b}, \mathrm{c}$ & 0.043312 & 0.053719 & 0.003221 & 0.018744 \\
\hline & & Total a & 0.564003 & 0.585575 & 0.346446 & 0.420407 \\
\hline & & Total b & 0.471494 & 0.452143 & 0.240852 & 0.301073 \\
\hline & & Total c & 0.449102 & 0.46872 & 0.680349 & 0.589778 \\
\hline \multirow[t]{10}{*}{4.8} & $\mathrm{C} \cup(\mathrm{A} \cup \mathrm{B})$ & $a$ & 0.263494 & 0.252102 & 0.159958 & 0.203157 \\
\hline & & $\mathrm{b}$ & 0.203832 & 0.228044 & 0.192184 & 0.204235 \\
\hline & & $\mathrm{c}$ & 0.306428 & 0.283046 & 0.650052 & 0.551342 \\
\hline & & $\mathrm{a}, \mathrm{b}$ & 0.036912 & 0.100707 & 0.02478 & 0.013949 \\
\hline & & $\mathrm{a}, \mathrm{c}$ & -0.0387 & 0.005502 & 0.005002 & -0.0152 \\
\hline & & $\mathrm{b}, \mathrm{c}$ & 0.096906 & 0.085198 & 0.023515 & 0.050371 \\
\hline & & $\mathrm{a}, \mathrm{b}, \mathrm{c}$ & 0.192916 & 0.176347 & 0.036331 & 0.09667 \\
\hline & & Total a & 0.49556 & 0.499648 & 0.262282 & 0.34503 \\
\hline & & Total b & 0.446234 & 0.5045 & 0.221263 & 0.298149 \\
\hline & & Total c & 0.462113 & 0.427209 & 0.617711 & 0.574802 \\
\hline \multirow[t]{4}{*}{4.9} & & $\mathrm{a}$ & & & & \\
\hline & Dienes) & & 0.283856 & 0.313245 & 0.242735 & 0.797505 \\
\hline & & $\mathrm{b}$ & 0.165168 & 0.142419 & 0.231828 & 0.031042 \\
\hline & & $\mathrm{c}$ & 0.22222 & 0.347252 & 0.268512 & 0.012824 \\
\hline
\end{tabular}




\begin{tabular}{|c|c|c|c|c|c|c|}
\hline Number & Exp/Ope & & Zadeh & Probabilístico & GMBCL & AMBCL \\
\hline & & $\mathrm{d}$ & 0.213386 & 0.18307 & 0.209362 & 0.046623 \\
\hline & & $a, b$ & 0.087152 & 0.105464 & 0.117102 & 0.031059 \\
\hline & & $\mathrm{a}, \mathrm{c}$ & -0.0937 & 0.000754 & $1.57 \mathrm{E}-05$ & 0.018705 \\
\hline & & $\mathrm{a}, \mathrm{d}$ & -0.111 & -0.0288 & -0.0169 & -0.0176 \\
\hline & & $b, c$ & -0.0282 & -0.0113 & -0.00648 & 0.016382 \\
\hline & & $\mathrm{b}, \mathrm{d}$ & -0.0748 & -0.038 & -0.0133 & -0.00637 \\
\hline & & $\mathrm{c}, \mathrm{d}$ & 0.058726 & 0.034203 & 0.090781 & 0.038898 \\
\hline & & $\mathrm{a}, \mathrm{b}, \mathrm{c}$ & 0.137813 & 0.033273 & 0.00617 & 0.019345 \\
\hline & & $a, b, d$ & 0.095048 & 0.050828 & 0.016845 & 0.019177 \\
\hline & & $\mathrm{a}, \mathrm{c}, \mathrm{d}$ & 0.182178 & 0.038564 & 0.01509 & 0.02481 \\
\hline & & $\mathrm{b}, \mathrm{c}, \mathrm{d}$ & 0.076229 & 0.028524 & 0.004181 & -0.00116 \\
\hline & & $\mathrm{a}, \mathrm{b}, \mathrm{c}, \mathrm{d}$ & -0.214 & -0.199 & -0.166 & -0.0312 \\
\hline & & Total a & 0.442383 & 0.406409 & 0.331562 & 0.870161 \\
\hline & & Total b & 0.284484 & 0.247544 & 0.306181 & 0.038611 \\
\hline & & Total c & 0.512543 & 0.374604 & 0.302421 & 0.08643 \\
\hline & & Total d & 0.370604 & 0.199758 & 0.270913 & 0.099029 \\
\hline \multirow[t]{19}{*}{4.10} & $(\mathrm{~A} \rightarrow \mathrm{B}) \cup(\mathrm{C} \rightarrow \mathrm{D})$ & $\mathrm{a}$ & 0.261604 & 0.23575 & 0.382687 & 0.665351 \\
\hline & & $\mathrm{b}$ & -0.0236 & 0.06862 & 0.15714 & 0.107719 \\
\hline & & $\mathrm{c}$ & 0.303188 & 0.257178 & 0.330495 & 0.040158 \\
\hline & & $\mathrm{d}$ & 0.052562 & 0.097066 & 0.144024 & 0.053299 \\
\hline & & $\mathrm{a}, \mathrm{b}$ & 0.075827 & 0.082409 & 0.048146 & 0.093287 \\
\hline & & $\mathrm{a}, \mathrm{c}$ & 0.060587 & -0.00122 & -0.0167 & 0.030302 \\
\hline & & $\mathrm{a}, \mathrm{d}$ & 0.091974 & 0.050276 & 0.02635 & 0.040716 \\
\hline & & $\mathrm{b}, \mathrm{c}$ & 0.047421 & 0.066142 & 0.007234 & -0.0128 \\
\hline & & $\mathrm{b}, \mathrm{d}$ & 0.043875 & 0.042371 & 0.018611 & 0.014562 \\
\hline & & $\mathrm{c}, \mathrm{d}$ & 0.102427 & 0.178072 & 0.09498 & 0.066585 \\
\hline & & $\mathrm{a}, \mathrm{b}, \mathrm{c}$ & 0.028798 & 0.004988 & 0.002305 & -0.0253 \\
\hline & & $\mathrm{a}, \mathrm{b}, \mathrm{d}$ & -0.0546 & -0.0427 & -0.016 & -0.0299 \\
\hline & & $\mathrm{a}, \mathrm{c}, \mathrm{d}$ & -0.0441 & -0.0215 & -0.0105 & -0.0412 \\
\hline & & $\mathrm{b}, \mathrm{c}, \mathrm{d}$ & 0.019506 & 0.00709 & -0.0145 & -0.00117 \\
\hline & & $\mathrm{a}, \mathrm{b}, \mathrm{c}, \mathrm{d}$ & 0.03452 & -0.0246 & -0.154 & -0.00159 \\
\hline & & Total a & 0.605337 & 0.616625 & 0.489322 & 0.769181 \\
\hline & & Total b & 0.188275 & 0.26359 & 0.287946 & 0.245881 \\
\hline & & Total c & 0.341802 & 0.355805 & 0.311698 & 0.027045 \\
\hline & & Total d & 0.109947 & 0.22464 & 0.190027 & 0.092092 \\
\hline \multirow{15}{*}{4.11} & & & & & & \\
\hline & $(\mathrm{A} \rightarrow \mathrm{B}) \cap(\mathrm{C} \rightarrow \mathrm{D})_{(\text {zadeh })}$ & $\mathrm{a}$ & 0.231976 & 0.237428 & 0.281553 & 0.241063 \\
\hline & & $\mathrm{b}$ & 0.190587 & 0.283548 & 0.288661 & 0.246261 \\
\hline & & $\mathrm{c}$ & 0.187957 & 0.231327 & 0.208401 & 0.216275 \\
\hline & & d & 0.234385 & 0.272059 & 0.231103 & 0.245636 \\
\hline & & $a, b$ & 0.076433 & 0.057977 & 0.053835 & 0.058751 \\
\hline & & $\mathrm{a}, \mathrm{c}$ & -0.0558 & -0.00451 & 0.002546 & -0.013 \\
\hline & & $\mathrm{a}, \mathrm{d}$ & -0.04 & -0.0111 & -0.0151 & -0.0177 \\
\hline & & $b, c$ & -0.0376 & -0.0301 & -0.0193 & -0.0294 \\
\hline & & $\mathrm{b}, \mathrm{d}$ & -0.0864 & -0.0322 & -0.0343 & -0.0544 \\
\hline & & $\mathrm{c}, \mathrm{d}$ & 0.070462 & 0.052749 & 0.061806 & 0.061263 \\
\hline & & $\mathrm{a}, \mathrm{b}, \mathrm{c}$ & 0.111077 & 0.036722 & 0.015905 & 0.049118 \\
\hline & & $\mathrm{a}, \mathrm{b}, \mathrm{d}$ & 0.119889 & 0.040062 & 0.029696 & 0.065921 \\
\hline & & $\mathrm{a}, \mathrm{c}, \mathrm{d}$ & 0.103489 & 0.023213 & 0.013796 & 0.04611 \\
\hline & & $\mathrm{b}, \mathrm{c}, \mathrm{d}$ & 0.130488 & 0.027506 & 0.024288 & 0.073259 \\
\hline
\end{tabular}




\begin{tabular}{|c|c|c|c|c|c|c|}
\hline Number & Exp/Ope & & Zadeh & Probabilístico & GMBCL & AMBCL \\
\hline & & $\mathrm{a}, \mathrm{b}, \mathrm{c}, \mathrm{d}$ & -0.237 & -0.185 & -0.143 & -0.189 \\
\hline & & Total a & 0.281111 & 0.279264 & 0.30884 & 0.272453 \\
\hline & & Total b & 0.385988 & 0.320069 & 0.323943 & 0.322273 \\
\hline & & Total c & 0.390578 & 0.313123 & 0.293448 & 0.34739 \\
\hline & & Total d & 0.35223 & 0.275025 & 0.271005 & 0.296044 \\
\hline \multirow{20}{*}{4.12} & & & & & & \\
\hline & $(\mathrm{A} \rightarrow \mathrm{B}) \cup(\mathrm{C} \rightarrow \mathrm{D})$ (zadeh) & $\mathrm{a}$ & 0.182626 & 0.086026 & 0.311161 & 0.269396 \\
\hline & & $\mathrm{b}$ & 0.11192 & 0.114027 & 0.20911 & 0.16836 \\
\hline & & $\mathrm{c}$ & 0.129038 & 0.109188 & 0.257837 & 0.220778 \\
\hline & & $\mathrm{d}$ & 0.139158 & 0.123941 & 0.247912 & 0.212391 \\
\hline & & $a, b$ & 0.03328 & 0.039173 & 0.012358 & 0.028326 \\
\hline & & $\mathrm{a}, \mathrm{c}$ & 0.02403 & 0.019306 & -0.0187 & -0.00367 \\
\hline & & $\mathrm{a}, \mathrm{d}$ & 0.1241 & 0.077578 & 0.022015 & 0.057059 \\
\hline & & $\mathrm{b}, \mathrm{c}$ & 0.103729 & 0.127638 & 0.031043 & 0.055845 \\
\hline & & $\mathrm{b}, \mathrm{d}$ & 0.067179 & 0.080926 & 0.060865 & 0.070436 \\
\hline & & $\mathrm{c}, \mathrm{d}$ & 0.107199 & 0.163177 & 0.032206 & 0.06055 \\
\hline & & $a, b, c$ & 0.016798 & -0.00696 & -0.00267 & -0.00326 \\
\hline & & $\mathrm{a}, \mathrm{b}, \mathrm{d}$ & -0.0622 & -0.0219 & -0.0203 & -0.0409 \\
\hline & & $\mathrm{a}, \mathrm{c}, \mathrm{d}$ & -0.0437 & 0.012185 & -0.00218 & -0.0215 \\
\hline & & $\mathrm{b}, \mathrm{c}, \mathrm{d}$ & -0.0187 & 0.059308 & -0.0229 & -0.0386 \\
\hline & & $\mathrm{a}, \mathrm{b}, \mathrm{c}, \mathrm{d}$ & 0.085444 & 0.01641 & -0.118 & -0.0352 \\
\hline & & Total a & 0.475123 & 0.549742 & 0.353606 & 0.400101 \\
\hline & & Total b & 0.266353 & 0.294936 & 0.275679 & 0.257706 \\
\hline & & Total c & 0.198855 & 0.226506 & 0.299065 & 0.237715 \\
\hline & & Total d & 0.381258 & 0.37987 & 0.29558 & 0.329653 \\
\hline
\end{tabular}

\section{Result analysis}

The operator should show changes when data oscillates in order to be able to analyze the robustness, but at the same time, it is necessary that this oscillations are not too big. After analyzing all data in the table 2 using the expression defined in step four of our methodology, we can assure that Compensatory Fuzzy Logic group holds the most robust operators and the most robust of them all is GMBCL.

\section{Conclusions and future works}

The Sobol model and the Monte Carlo simulations are a powerful tool to analyze the robustness of the fuzzy operators. In the current research the most robust operators belong in the Compensatory Fuzzy Logic group. As future studies we propose to extend the analysis to other operators as well as to other expressions.

\section{References}

1) BOUCHET, A., ESPIN ANDRADE, R. A., PASTORE, J. I., BRUN, M. \& BALLARIN, V. 2010. A based arithmetic mean compensatory logic. ALIO-INFORMS Joint international meeting. Buenos Aires, Argentina.

2) CEBECI, U. 2009. Fuzzy AHP-based decision support system for selecting ERP systems in textile industry by using balanced scorecard. Expert Systems with Applications, 36, 8900-8909.

3) DUBOIS, D. \& PRADE, H. 1985. Review of fuzzy set aggregation connectives. Information sciences (36), 85-121.

4) ESPIN ANDRADE, R. A., MARX GÓMEZ, J., MAZCORRO TÉLLEZ, G. \& FERNÁNDEZ GONZÁLEZ, E. 2003. Compensatory logic: a fuzzy formative model for decision making. Congress of international association for fuzzy-set management and Economy. León, España.

5) GLEN, G. \& ISAACS, K. 2012. Estimating Sobol sensitivity indices using correlations. Environmental Modelling \& Software, 37, 157-166.

6) MERIGÓ, J. M. \& GIL-LAFUENTE, A. M. 2011. Fuzzy induced generalized aggregation operators and its application in multi-person decision making. Expert Systems with Applications, 38, 9761-9772.

7) PIEGAT, A. 2001. Fuzzy modeling and control. Physica-Verlag. 
8) SALTELli, A., ANDRES, T., CAMPOLONGO, F., CARIBONI, J., GATELLI, D., SAISANA, M. \& TARANTOLA, S. 2008. Global Sensitivity Analysis. The Primer. In: LTD, J. W. S. (ed.). The Atrium, Southern Gate, Chichester, West Sussex PO19 8SQ, England: John Wiley \& Sons Ltd.

9) SALTELLI, A., TARANTOLA, S., CAMPOLONGO, F. \& RATTO, M. 2004. Sensitivity analysis in practice : a guide to assessing scientific models. In: LTD, J. W. S. (ed.). The Atrium, Southern Gate, Chichester, West Sussex PO19 8SQ, England: John Wiley \& Sons Ltd.

10) WEI, G. \& ZHAO, X. 2012. Some induced correlated aggregating operators with intuitionistic fuzzy information and their application to multiple attribute group decision making. Expert Systems with Applications, 39, 2026-2034.

11) YANG, J. 2011. Convergence and uncertainty analyses in Monte-Carlo based sensitivity analysis. Environ. Modell. Softw. 26, 444e457.

12) YÜKSEL, İ. \& DAĞDEVIREN, M. 2010. Using the fuzzy analytic network process (ANP) for Balanced Scorecard (BSC): A case study for a manufacturing firm. Expert Systems with Applications, 37, 12701278.

13) ZADEH, L. A. 1973. Outline of a new approach to the analysis of complex systems and decision processes. Transactions on systems, man, and cybernetics, 36-38.

14) ZANDI, F. \& TAVANA, M. 2012. A fuzzy group multi-criteria enterprise architecture framework selection model. Expert Systems with Applications, $39,1165-1173$. 